doi:10.17659/01.2020.0016

Journal of Case Reports 2020;10(1):60-63

\title{
Is There a Role for Bone Marrow Biopsy In The Diagnosis of Masked Polycythemia Vera with Splanchnic Vein Thrombosis?
}

\author{
Maria Cristina Scamuffa, Roberto Latagliata, Luisa Bizzoni, Maria Lucia De Luca, Federica Falco, Sofia \\ Chiatamone Ranieri, Massimo Breccia, Robin Foà, Marco Vignetti, Stefania Trasarti \\ Division of Hematology, Department of Precision and Translational Medicine, Sapienza University, Rome, Italy.
}

\begin{abstract}
Corresponding Author:
Dr Stefania Trasarti

Email: trasarti@bce.uniroma1.it

This is an Open Access article distributed under the terms of the Creative Commons Attribution License (creativecommons.org/ licenses/by/3.0).
\end{abstract}

Received : December 12, 2019

Accepted : March 14, 2020

Published : March 25, 2020

\begin{abstract}
Background: Philadelphia-negative chronic myeloproliferative neoplasms are the most common underlying prothrombotic disorders found in patients with splanchnic vein thromboses. A sizeable proportion of patients with splanchnic vein thromboses carry a Jak2-V617F mutation, despite the absence of overt signs of myeloproliferative neoplasm. Case Report: We report a case of a 46-year-old male presenting with splenomegaly and anemia. Subsequently, due to the appearance of abdominal pain, thrombosis of the portal vein, intra-hepatic portal branches and splenic vein were found. The simultaneous presence of splenomegaly and splanchnic vein thromboses prompted a search for an underlying myeloproliferative neoplasm and a diagnosis of splanchnic vein thromboses from a "masked polycythemia vera" was posed. Conclusion: This uncommon case highlights the importance of an early myeloproliferative neoplasms screening when a splanchnic vein thromboses is diagnosed.
\end{abstract}

Keywords: Myeloproliferative Disorders, Polycythemia Vera, Portal Vein, Splenic Vein, Splenomegaly, Thrombosis.

\section{Introduction}

Polycythemia vera (PV), essential thrombocythemia (ET), idiopathic myelofibrosis (IM), known as Philadelphia-negative chronic myeloproliferative neoplasms (MPN), are the most common causes of splanchnic vein thrombosis (SVT) [1]. SVT refers to thrombotic formations in the portal venous system (PVT), hepatic venous system, splenic venous system or mesenteric venous system. In patients with SVT, there is an important activation of the coagulation cascade due to the presence of hereditary thrombophilic changes, or acquired as lupus anticoagulant or MPN. While the diagnosis of thrombophilia is relatively simple and accurate, the diagnosis of MPNs is often difficult in patients with a thrombosis of the portal axis [2].

A sizeable proportion of patients with SVT carry a Jak2-V617F mutation, despite the absence of overt signs of MPN. Many of these patients also share bone marrow morphology with hypercellularity and trilineage hyperplasia, conforming to the WHO criteria of PV, but hemoglobin $(\mathrm{Hb})$ levels are lower than those of the WHO threshold for a diagnosis of PV. This condition, together with other clinical conditions not reaching $\mathrm{Hb}$ levels for a full diagnosis of $\mathrm{PV}$, has been defined as "masked PV". To avoid misdiagnoses and consequent under treatment of "masked PV", the $\mathrm{Hb}$ threshold required as a criterion for $\mathrm{PV}$ diagnosis in the $\mathrm{WHO}$ 2016 classification has been lowered to $16.5 \mathrm{~g} / \mathrm{dL}$ in males and $16 \mathrm{~g} / \mathrm{dL}$ in females. However, some patients still escapes from a correct diagnosis, as documented by the case hereby described.

\section{Case Report}

A 46-years old Caucasian man was admitted in April 2017 for splenomegaly. Past medical history 
was unremarkable, except for hypertension and surgical intervention for varicocele. In the context of routine analyses for hypertension, he performed an abdominal ultrasound that revealed the presence of splenomegaly (DCL $21 \mathrm{~cm}$ ). At physical examination, the spleen was palpable $3 \mathrm{~cm}$ below the left costal margin.

Peripheral blood evaluation revealed a mild microcytic anemia: $\mathrm{Hb} 12.1 \mathrm{~g} / \mathrm{dL}$, hematocrit $39.9 \%$, mean corpuscular volume $69.2 \mathrm{fl}$, white blood cells $5.2 \times 10^{9} / \mathrm{L}$, neutrophils $3.2 \times 10^{9} / \mathrm{L}$, lymphocytes $1.1 \times 10^{9} / \mathrm{L}$, platelets $167 \times 10^{9} / \mathrm{L}$. Reticulocytes were $1.1 \%$, ferritin $24 \mathrm{ng} / \mathrm{mL}, \mathrm{LDH}$ $233 \mathrm{U} / \mathrm{L}$, total bilirubin $0.61 \mathrm{mg} / \mathrm{dL}$. Coagulation tests were in the normal range as well as the thrombophilia screening. Antibodies against hepatitis $\mathrm{B}$ and $\mathrm{C}$, and HIV, as well as screening for autoimmune disorders were all negative.

Microscopic evaluation of peripheral blood smears revealed the presence of several ellissocytes. An ultrasound of superficial lymph nodes was normal. The patient underwent a marrow needle aspirate, which was not evaluable for hypocellularity, while karyotype was normal. In view of a possible genetic hematologic disorder given the presence of ellissocytes, complete work-up for red blood cell inherited alterations was performed, including acidified glycerol lysis, osmotic fragility and thermogravimetry tests, but no erythrocyte membrane protein defects was identified. The presence of a genetic hematologic disease was therefore excluded. In July 2017, due to the appearance of abdominal pain, another abdominal ultrasound was carried out that documented a portal vein with a reduced flow and with a concomitant flow increase in the hepatic and splenic arteries. In order to exclude the presence of a lymphoproliferative disorder, a positron-emission computed tomography (PET-CT) examination was performed that demonstrated lack of visualization of the peri-hepatic and intra-hepatic portal system, a filiform splenic vein and an increased uptake of tracer at the spleen level in the absence of focal hyper-accumulation areas.
Furthermore, gastroenterologic evaluation detected the presence of grade F2 varices at the middle and lower third of esophagus. A diagnosis of pre-hepatic portal hypertension was therefore established. The patient started treatment with heparin 6000 IU/day and propranolol $40 \mathrm{mg} /$ day, as prophylaxis for bleeding from esophageal varices. One month later, in a repeated CT of the abdomen, a thrombosis of the portal vein with also an involvement of the right and left intra-hepatic portal branches [Fig. 1] as well as a thrombosis of the splenic vein with multiple perisplenic, splenorenal and perigastric collaterals were found. In the context of the spleen, the presence of a $6 \mathrm{~cm}$ hypodense area, possible expression of an ischemic lesion, was highlighted. In the suspect of a masked MPN, a Jak2-V617F screening was performed, which proved positive. The patient then underwent a bone biopsy, which showed hyper-cellular bone marrow with trilinear expansion in the absence of significant fibrosis: this histologic picture was consistent with PV and thus a diagnosis of SVT from a "masked PV" was posed.

Cytoreductive treatment with hydroxyurea (500 mg/day) was started. A year later, the patient is currently receiving hydroxyurea every other day, in the absence of any new thrombotic or hemorrhagic manifestations, with a peripheral blood count in the standard values except for mild thrombocytopenia (platelet $105 \times 10^{9} / \mathrm{L}$ at the last evaluation).

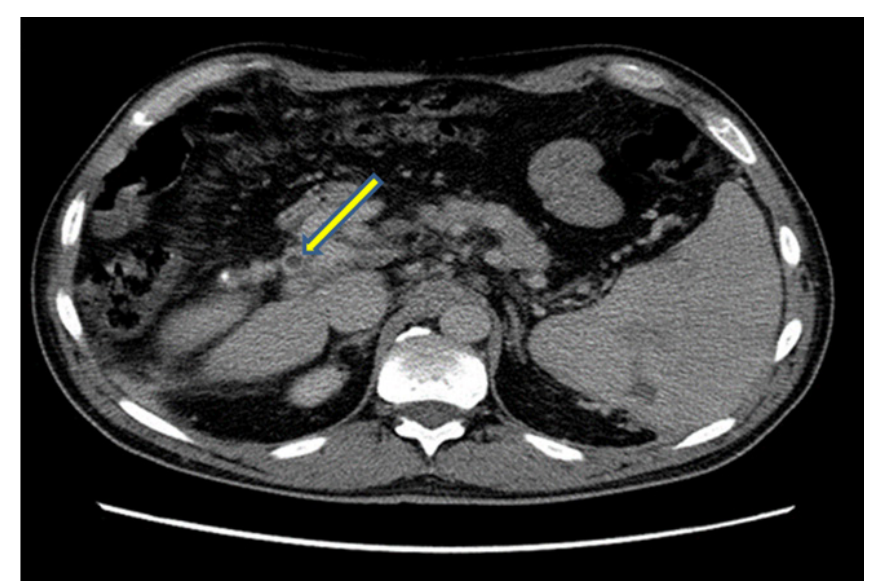

Fig.1: Thrombosis of the right portal branch. 


\section{Discussion}

MPN are the most common underlying prothrombotic disorders found in these patients [3]. The rate of mortality due to thrombosis of the hepatic veins is greater in patients diagnosed with MPN than in those who do not have an underlying MPN [3]. A systematic review of 24 studies found that Jak2-V617F conferred a significantly increased risk of SVT formation [4]. Megakaryocytes carrying a Jak2-V617F mutation show a greater chemotaxis along migration assays, as well as an increased pro-platelet formation in response to low thrombopoietin levels [5]. Moreover, red blood cells (RBC) also display increased adhesion to endothelium in PV patients by inducing phosphorylation of the RBC-adhesion receptor Lutheran/basal cell-adhesion molecule (Lu/BCAM), which enhances RBC binding to subendothelial laminin [6].

The strong clinical association between SVT and MPN is already recognized and has led to recommendations for an early MPN screening when a SVT is diagnosed [7]. SVT has an estimated prevalence of 5-10\% in PV and 9-13\% in ET [8], respectively, while it occurs less frequently in IM (0.6-1\%) [9]. An estimated $30 \%$ of patients with PVT and $40 \%$ of patients with BCS have an underlying MPN and a significant proportion of patients with SVT (15-17\%) test positive for Jak2-V617F mutations [7]. It is worth noting that some patients with SVT and an underlying MPN do not otherwise meet all WHO criteria for MPN. Indeed, a diagnosis of MPN (and particularly PV) is often difficult in these patients because of the spleen enlargement caused by portal hypertension, secondary pancytopenia, bleeding disorders and hyper-dilution [10]: all these factors can mask peripheral blood features (polyglobulia, leukocytosis and thrombocytosis) typical of MPN and used in the diagnostic evaluation according to the WHO criteria. Indeed, one study estimated that testing for Jak2-V617F can identify an additional
$30 \%$ of MPN patients who do not otherwise have overt evidence of disease [11]. These data reinforce the recommendation to include Jak2-V617F testing in the work-up of patients presenting with a newly diagnosed SVT.

A second step is clinically relevant in patients with SVT and Jak2-V617F mutation, in order to establish the single MPN entity, due to the different clinical and therapeutic approaches among PV, ET and IM. In contrast with peripheral blood features, bone marrow biopsy is largely independent from spleen enlargement, spleen sequestration and hemodilution, and is capable of discriminating between the different MPN entities. Thus, marrow biopsy is the tool of choice in these patients and should always be carried out. In our case, while the peripheral blood examination was unable to discriminate PV features notwithstanding the recent lowering of hemoglobin threshold in WHO 2016 criteria, marrow biopsy led us to the correct diagnosis of PV due to the peculiar evidence of trilinear hyperplasia without significant marrow fibrosis. It is worth noting that a correct diagnosis of PV in patients with SVT is becoming increasingly crucial, due to the possible use of ruxolitinib as second line, at variance from ET.

\section{Conclusion}

In MPN associated with SVT at onset, criteria usually employed in the diagnostic process of "typical" forms are not always specific. The strong association of Jak2-V617F mutation with SVT makes it an important first-line diagnostic test of screening for latent MPN. However, in all patients with SVT and Jak2-V617F mutation, a marrow biopsy is a second-line mandatory examination, in particular for a correct diagnosis of PV and a consequent therapeutic tailored approach.

Contributors: M.C.Scamuffa: literature review, development of manuscript; S.Trasarti: patient management and diagnosis provided, oversaw development of manuscript, edited manuscript; R.Latagliata, M.Breccia critical inputs into the manuscript; M. Breccia: supervised the patient care, oversaw 
development of manuscript, edited manuscript; R.Foà manuscript editing. S.Trasarti will act as a study guarantor. All authors approved the final version of this manuscript and are responsible for all aspects of this study.

Funding: None; Competing interests: None stated.

\section{References}

1. Yonal I, Pinarbası B, Hindilerden F, Hancer VS, Nalcaci $\mathrm{M}$, Kaymakoglu S, et al. The clinical significance of JAK2V617F mutation for Philadelphia-negative chronic myeloproliferative neoplasms in patients with splanchnic vein thrombosis. J Thromb Thrombolysis. 2012;34:388396.

2. Karaköse S, Oruç N, Zengin M, Akarca US, Ersöz G. Diagnostic value of the JAK2 V617F mutation for latent chronic myeloproliferative disorders in patients with Budd-chiari syndrome and/or portal vein thrombosis. Turk J Gastroenterol. 2015;26:42-48.

3. How J, Zhou A, Oh ST. Splanchnic vein thrombosis in myeloproliferative neoplasms: pathophysiology and molecular mechanisms of disease. Ther Adv Hematol. 2017;8:107-118.

4. Dentali F, Squizzato A, Brivio L, Appio L, Campiotti $\mathrm{L}$, Crowter M, et al. JAK2V617F mutation for the early diagnosis of $\mathrm{Ph}$ - myeloproliferative neoplasms in patients with venous thromboembolism: a meta-analysis. Blood. 2009;113:5617-5623.

5. Hobbs C, Manning H, Bennet C, Vasquez L, Severin S, Brain L, et al. JAK2V617F leads to intrinsic changes in platelet formation and reactivity in a knock-in mouse model of essential thrombocytemia. Blood. 2013;122:3787-3797.

6. De Grandis M, Cambot M, Wautier MP, Cassinat B, Chomienne $\mathrm{C}$, Colin $\mathrm{Y}$, et al. JAK2V617F activates $\mathrm{Lu} / \mathrm{BCAM}$-mediated red cell adhesion in polycythemia vera through an EpoR-indipendent Rap1/Akt pathway. Blood. 2013;121:658-665.

7. Smalberg J, Arends L, Valla D, Kiladjian J, Janssen H, Leebeek F. Myeloproliferative neoplasms in BuddChiari syndrome and portal vein thrombosis: a metaanalysis. Blood. 2012;120:4921-4928.

8. De Stefano V, Za T, Rossi E, Vannucchi A, Ruggeri $\mathrm{M}$, Elli E, et al. Recurrent thrombosis in patient with polycythemia vera and essential thrombocythemia: incidence, risk factors and effect of treatments. Haematologica. 2008;93:372-380.

9. Barbui T, Carobbio A, Cervantes F, Vannucchi A, Guglielmelli P, Antonioli E, et al. Thrombosis in primarymyelofibrosis: incidence and riskfactors. Blood. 2010;115:778-782.

10. Fama A, Rago A, Gioiosa F, Marzano C, Latagliata R, Mammì C, et al. Sindrome di Budd-Chiari e trombosidellevenesplancniche: neoplasiemieloproliferativemescherate e mutazione di JAK2V617F. Clin Ter. 2010;161:169-171.

11. De Stefano V, Vannucchi AM, Ruggeri M, Cervantes F, Alvarez-Larran A, Iurlo A, et al. Splanchnic vein thrombosis in myeloproliferative neoplasms: risk factors for recurrences in a cohort of 181 patients. Blood Cancer J. 2016;6:e493. 